\title{
Citicoline as a suggested novel adjuvant for painful diabetic polyneuropathy
}

\author{
Dico Gunawijaya, I Putu Eka Widyadharma, Ida Ayu Sri Wijayanti \\ Deparment of Neurology, Udayana University/Sanglah Hospital, Denpasar, Bali, Indonesia
}

\begin{abstract}
The purpose of this paper is to review the effectiveness of citicoline as suggested adjuvant therapy for painful diabetic polyneuropathy based on evidences.

Pain is one of the most common symptoms that make patients consult with a doctor, especially chronic pain. One of the examples is painful diabetic polyneuropathy, which prevalence is increasing by global development. Diabetic polyneuropathy is the most common cause of neuropathic pain caused by long-term complications of microangiopathy. Affect not only individual socioeconomic status but also the psychological aspect of the patient. Neuropathic pain is one of the most common causes of long-term disability. Some medicines already recommended as the drug of choice, but not all of them give maximum results. Adjuvant neuroprotector therapy is often considered to help manage painful diabetic polyneuropathy, such as citicoline, which has been proven in some studies. Painful diabetic polyneuropathy is very challenging because of its pathophysiology, which has not fully understood. The different mechanism of pain sensation is still unknown but it is thought that the oxidative stress after microangiopathy triggers the discharge of abnormal load from damaged neurons. Some analgetics have not given the expected result.

Conclusion. Citicoline may be suggested as adjuvant therapy based on evidences with animal subject, but further studies with human subject are still needed.
\end{abstract}

Keywords: neuropathic pain, diabetic polyneuropathy, citicoline

\author{
Abbreviations \\ EPT - extensor postural thrust; \\ SFI - sciatic functional index; \\ EMG - electromyography \\ MMP - matrix metalloproteinase; \\ TIMPs - tissue inhibitors of metalloproteinases
}

\section{INTRODUCTION}

Neuropathic pain is one of the most common causes of long-term disability. This condition arises due to damage to neurons that deliver stimulation, both in the central and peripheral nervous systems $[1,2]$. Diabetic polyneuropathy is the most common cause of neuropathic pain caused by long-term complications of microangiopathy. Studies in the United States showed that $47 \%$ of diabetic patients upon diagnosis was already accompanied by neuropathy, with more than half of these being distal symmetric polyneuropathy $[3,4]$. Neuropathic pain in diabetic polyneuropathy is found in $16 \%$ to $26 \%$ of patients. The disease's duration is supposedly related to the development and progression of diabetic polyneuropathy, which contributes to the onset of neuropathic pain in patients with diabetes mellitus [5].

The risk factors for neuropathic pain due to diabetic polyneuropathy are still unknown compared to painless diabetic neuropathy. Yet, they are thought to be due to chronic hyperglycemia as a significant risk factor [6]. The factors that cause 
the development of diabetic polyneuropathy are not fully understood. Generally, these factors are described into two types, namely metabolic and vascular factors [7]. Prolonged hyperglycemia results in increased activity of the polyol pathway, formation of free radicals and activation of protein kinase C. Activation of these pathways results in a lack of vasodilation, resulting in decreased blood flow to the nerves, resulting in diabetic polyneuropathy [8]. Hyperglycemia also stimulates the production of reactive oxygen species, which cause vascular endothelial damage and neutralize nitric oxide. This can prevent microvascular vasodilation. The mechanism can be through thickening of the basal membrane, thrombocytes in intraneural arterioles, an increase in platelet aggregation; reduced nerve blood flow and increased vascular resistance; and axonal stasis, swelling, and demyelination of the nerves due to acute ischemia [9].

Symptoms of neuropathic pain are experienced by half of the patients with diabetic polyneuropathy, with chronic peripheral sensorimotor neuropathy as the most commonly found. Generally, pain is bilaterally symmetrical, progresses slowly, and starts from the distal area [3]. Symptoms can also be asymmetrical or focal and multifocal, or symmetrical or diffuse [6]. Pain sensations vary between patients; some might describe the pain, such as burning, shock, or needling. The different mechanism of pain sensation is still unknown, but it is thought that the oxidative stress after microangiopathy triggers the discharge of abnormal load from damaged neurons. The intensity of pain in most patients is moderate to severe, and it is not uncommon to find hyperalgesia and allodynia [10].

\section{MANAGEMENT OF PAINFUL DIABETIC NEUROPATHY}

Many aspects need to be engaged in controlling painful diabetic polyneuropathies, such as glycemic control, general care, and symptomatic treatment. Glycemic control and $\mathrm{HbA1c}$ routine monitoring are the basics that need to be done; it is suggested that the HbAlc level is below $6 \%-7 \%$. Other metabolic factors, such as hemoglobin, albumin, and lipid, also need to be controlled. [9] Keeping skin hygiene and avoiding any injury of the lower extremity is necessary to prevent com- plications such as infection, gangrene, and amputation [11].

Painful diabetic polyneuropathy has its challenge because of its pathophysiology, which has not fully understood, and some analgetics have not given the expected result. Several drugs, standalone or combination, have been proven to significantly reduce neuropathic pain compared with placebo in some clinical trials, but these analgetics are still not adequate for most patients. In daily practice, there is rarely a single drug capable of treating painful diabetic polyneuropathy. Even so, treatment of pain usually starts with antidepressants or anticonvulsant medication. The dose can be increased until the maximum dose or if side effect starts to arise [7].

Pregabalin is a type of anticonvulsant known to overcome pain in diabetic polyneuropathy, with the recommended dosage of 300-600 $\mathrm{mg} /$ day. Some guidelines also recommend gabapentin 9003,600 mg/day [9]. Antidepressants are also an option in the treatment of painful diabetic polyneuropathy, which are serotonin-norepinephrine reuptake inhibitor group and tricyclic antidepressants. Duloxetine and venlafaxine, two types of SNRIs, have been approved in the United States to treat painful diabetic polyneuropathy [7]. The recommended dosage is $60-120 \mathrm{mg} /$ day for duloxetine and 75-225 mg/day for venlafaxine. Amitriptyline is proven to be as effective as gabapentin in a meta-analysis study, with a dose of $25-100 \mathrm{mg} /$ day [9]. Opioids are recommended as a second or third-line treatment for painful diabetic polyneuropathy. One study reported tramadol effectiveness for significantly increased scores on physical and social functioning in patients with painful diabetic polyneuropathy. Morphine has also been shown to be effective in reducing the average daily pain score associated with diabetic polyneuropathy [8]. Other treatments that can be given include topical capsaicin, lidocaine patches, and isosorbide dinitrate $[7,9]$.

\section{REVIEW OF CITICOLINE}

Citicoline or cytidine 5-diphosphocholine (CDP-choline) is a nucleotide compound which consists of choline, cytosine, pyrophosphate, and ribose that can be seen in Figure 1 [12]. Two large parts that form citicoline are cytidine and choline, 
which are connected with the diphosphate chain [13]. Those components are essential in the cell membrane's phospholipid biosynthesis, such as phosphatidylcholine and biosynthesis of one primary neurotransmitter acetylcholine. Adequate synthesis of phospholipid is necessary for cell membranes to keep the cell's function [14].

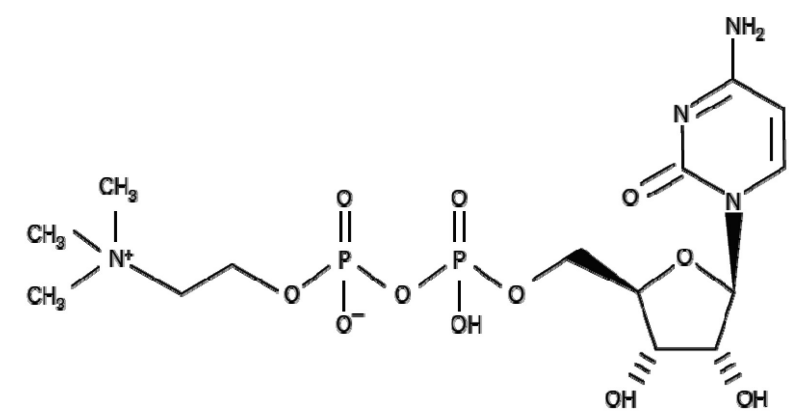

FIGURE 1. Chemical structure of citicoline [12]

\section{Pharmacokinetic}

Oral citicoline is hydrophilic and has a biphasic peak plasma level, one hour, and 24-hour post-administration. Oral citicoline absorption is quick, and its bioavailability is more than $90 \%$, with excretion in feces less than 1\%. [13-15]. Citicoline is hydrolyzed in the small intestine and liver, with products such as choline and cytidine. Both of these precursor compounds are distributed in the body's tissues, including the central nervous system, and are re-synthesized into citicoline by the enzyme cytidine-triphosphate-phosphocholine cystidylyl transferase. Although only a small amount of citicoline passes through the bloodbrain barrier as choline and cytidine, its use in the central nervous system is very efficient. This precursor level will increase to $2 \%$ if citicoline is given intravenously [15].

A metabolite of citicoline, which is choline and cytidine, re-synthesized endogenous citicoline, which then will help the formation of neuron phospholipid [14]. Choline, which is classified in the B group of vitamins, plays a role in three metabolic processes, namely the synthesis of phospholipids through phosphorylcholine, acetylcholine synthesis, and oxidation of betaine as methyl donors. After being converted to uridine, cytidine is a major component of nucleic acid synthesis and synthesis of membrane and glycosylation components [12]. Cytidine will later undergo cytoplasmic conversion to cytidine triphosphate. In citicoline metabo- lism, choline will be phosphorylated by the enzyme choline and produce phosphorylcholine. This phosphorylcholine combines with cytidine triphosphate to form citicoline. Excretion is mainly through the lungs and kidneys, with an elimination half-life of 56 hours for $\mathrm{CO} 2$ and 71 hours for urine $[12,15]$.

\section{Pharmacodynamic}

Citicoline has some neuroprotector mechanisms. As a phospholipid precursor, citicoline maintains the integrity of damaged neuron cell membranes, for example, ischemia. The ischemic condition will lead to the destruction of cell membranes, which triggers pro-inflammatory cascade, causing free fatty acid, arachidonic acid, and glycerol buildup. Formed toxin metabolites such as prostaglandin, thromboxane and free radicals will worsen the neuron cell's damage. Administration of citicoline is proven to reduce free fatty acids, arachidonic acid, and other toxin metabolites, thereby preventing worsening conditions due to inflammatory processes and preventing organ damage due to endotoxins [14]. Citicoline inhibits phospholipid catabolism (autocanibalism) and has a protective effect on ATPase membranes and enzymes, which are essential for nerve metabolism, specifically succinyl dehydrogenase and citrate synthetase [12]. Citicoline maintains cardiolipin by inhibiting the activity of phospholipase-A2, which is a component of the mitochondrial membrane. Cardiolipin is an important regulator in maintaining the mitochondrial function, thus enabling cell healing and regeneration [13].

\section{PATHOLOGY OF PAINFUL DIABETIC POLYNEUROPATHY, EVIDENCE OF CITICOLINE EFFECTIVENESS ON PERIPHERAL NERVE LESION, AND THEIR SUGGESTED CORRELATION}

Diabetic polyneuropathy that affect sensorimotor system can manifest as pain, paresthesia, or hypo-/anesthesia $[3,4,10]$. Mechanism of these different sensation disturbances still could not be distinguished, but it is believed to be multifactorial. Abnormal discharge or ectopic impulse firing in the peripheral nociceptor system can cause painful sensations due to hyperexcitability of neural membrane. Microangiopathy that leads to oxidative 
stress is suggested as the main pathological process in diabetic polyneuropathy [16]. Thickening of basal membrane, endothelial and smooth muscle edema, and excessive platelet aggregation are seen in vascular abnormality. Evidence of inflammation process also has been proved as pathological mechanism in diabetic polyneuropathy [6]. Painful diabetic polyneuropathy is one of peripheral nerve lesion that commonly found in general population. Beside the glycemic control, author suggested that neuroprotector administration could be beneficial in neural regeneration, although there is still lack of evidence with human sample. These are some evidence that showed the effectiveness of citicoline in peripheral nerve lesion, with or without neuropathic pain.

Emril et al. reported citicoline administration inhibits neuropathic pain and accelerate motoric improvement in 4 weeks on animal trial with sciatic nerve lesion. The subjects of this study were exposed to trauma to the sciatic nerve of the right medial region of the femur, then given a gelatin sponge containing citicoline $0.4 \mathrm{ml}(100 \mu \mathrm{mol} / 1)$, citicoline $0.8 \mathrm{ml}(100 \mu \mathrm{mol} / 1)$, and $0.4 \mathrm{ml}$ of saline as control. The evaluation was carried out four weeks post-trauma, which made use of Von-Frey filaments with a threshold of $100 \mathrm{~g}$ for the assessment of neuropathic pain and the extensor postural thrust (EPT) test for the evaluation of motor function. Sciatic functional index (SFI) was assessed by analyzing the image of the animal's paw when walking. The results showed the citicoline $0.4 \mathrm{ml}$ subject group had a lower pain percentage than the control group (23.53\% versus $53.33 \%$ ). The EPT test also showed better motor improvement. In contrast, the portion of pain was found to be higher (66.67\%) and poor EPT test in the $0.8 \mathrm{ml}$ citicoline subject group, probably due to the suppression of a sponge with a higher volume. SFI analysis did not show significant differences between the three groups. From this study, it can be concluded temporarily that citicoline has the potential to help the recovery of peripheral nerves through its mechanism to help regenerate axons [17].

Another study conducted by Ozay et al. showed improvement of peripheral nerve lesion on animal trials after citicoline administration. Transection of the right sciatic nerve and the epidural suture was performed on subjects. The subjects were given topical agents and divided into two groups: the group with citicoline $0.4 \mathrm{ml}$ and group with saline $0.4 \mathrm{ml}$. After 12 weeks, functional recovery appeared to be significantly better on the group with citicoline compared with the control group. The citicoline subject group significantly showed a more significant number of axons and mean axon diameters and a better histological organization of axons. Besides, the quantity of postoperative scarring was comparatively lower than in the control group. The amplitude of the action potential was assessed with electromyography (EMG), which showed significantly better results in the subject group with citicoline [18]. The findings of Liu et al. also showed the same results as the sciatic nerve transection method. The subject group with citicoline $(80 \mathrm{mg} / \mathrm{ml})$ showed good EMG results three months later and improved edema and nerve adhesion [19].

A study by Aslan et al. used an animal trial that underwent sciatic nerve transection, then divided into two groups: epineural repair post-transection or three days afterward. Subjects received a topical regimen of $0.4 \mathrm{ml}$ of saline or $100 \mu \mathrm{M}$ citicoline, cytidine, choline, or cytidine + choline. SFI analysis in both groups (immediate repair and delayed repair) showed a gradual increase at four, eight, and 12 weeks post-transection. The group that received the citicoline or cytidine + choline regimen had a significantly higher percentage of SFI increase in all three periods, increased the number of axons to $50 \%$, and a better axon arrangement. In the immediate repair group with citicoline or cytidine + choline showed the low formation of scar tissue and low levels of adhesion with the surrounding tissue. There were no significant improvements in SFI and histology in the cytidine or choline regimens [20].

The method of sciatic nerve transection also performed by Caner et al., but the difference was the intervention was administered through intraperitoneal (systemic). Subject animals were divided into five groups: the group with saline $1 \mathrm{ml} / \mathrm{kg}$ as control and other subject groups with saline plus citicoline, cytidine, choline, and cytidine choline $600 \mathrm{mmol} / \mathrm{kg}$. SFI improvement is reportedly better in the group with citicoline, cytidine, choline, and cytidine choline in 8-12 weeks. EMG analysis showed better regeneration than the control group, which were $228 \%$ citicoline, $168 \%$ choline, and $221 \%$ cytidine choline compared to controls. The 
number and density of axons also increased significantly in these three groups. However, the cytidine subject group did not show any changes in SFI, EMG, and histology. Only the subject group of citicoline showed a low level of adhesion of nerves to the surrounding tissue [21].

Gundogdu et al. reported intraperitoneal administration of $600 \mathrm{mg} / \mathrm{kg}$ of citicoline was able to reduce MMP activity and increase the formation of TIMPs to stimulate axon regeneration compared to control groups (saline $1 \mathrm{ml} / \mathrm{kg}$ ). MMP-2 and MMP-9 concentrations increase after neuronal trauma. During tissue healing, the extracellular matrix will indeed be destroyed to reorganize the cells in the matrix. However, excessive destruction inhibits regeneration itself because there will be damage to the blood-neuron barrier that triggers macrophage and neutrophil infiltration. TIMP-1 and TIMP-3 are important proteins that protect from excessive destruction by indirectly binding to MMP [22,23].

A similar study by Kaplan et al. concluded that intraperitoneal citicoline has a dose-dependent effect in axon regeneration in animals that underwent sciatic nerve transection and primary anastomosis. Subjects divided into categories based on an intervention, which were citicoline $300 \mu \mathrm{mol} /$ $\mathrm{kg}$ (C-300), $600 \mu \mathrm{mol} / \mathrm{kg}$ (C-600), and $900 \mu \mathrm{mol} /$ $\mathrm{kg}$ (C-900), with a control group of saline $2 \mathrm{ml}$. C-600 group showed significantly increase SFI on 12 weeks postoperative, while C-900 group on 8 and 12 weeks postoperative. Analysis of the action potential latency in the C-600 and C-900 EMG groups was lower than in the control group. Still, there was no significant difference in amplitude between the three citicoline groups. Loose connec-

\section{REFERENCES}

1. Berry PH, Covington EC, Dahl JL, Katz JA, Miaskowski C. Pain: Current Understanding of Assessment, Management, and Treatments. National Pharmaceutical Council. 2011:3-18.

2. Purba JS, Aninditha T. Nyeri Neuropatik. Dalam: Departemen Neurologi FKUI-RSCM. Buku Ajar Neurologi. Tangerang: Perkisa. 2017;2(11):598-608.

3. Juster-Switlyk K, Smith AG. Updates in diabetic peripheral neuropathy. F1000Res. 2016.

4. DiBonaventura MD. The prevalence of probable neuropathic pain in the US: results from a multimodal general-population health survey. Journal of Pain Research. 2017;10:2525-38.

5. Katulanda P, Ranasinghe P, Jayawardena R, Constantine GR, Sheriff MHR, Matthews DR. The prevalence, patterns, and predictors of diabetic peripheral neuropathy in a developing country. Journal of Diabetology \& Metabolic Syndrome. 2012;4:21. tive tissue appeared around the wound 12 weeks postoperatively in the citicoline group, while the control group produced solid connective tissue. The mean axon density and the number of myelinated axons were significantly higher in the C-900 group than in the other groups [24].

A clinical trial in humans has been conducted to assess ligustrazine and citicoline's efficacy in the treatment of peripheral diabetic polyneuropathy. 300 subjects were randomly divided into three therapy groups, namely the ligustrazine and citicoline groups (group A), ligustrazine monotherapy (group B), and citicoline monotherapy (group C). After four weeks of therapy, improvements in blood sugar and cholesterol levels appeared in all three groups but did not differ significantly. Symptomatic integral and EMG showed better improvement in group A three months post-treatment $(\mathrm{p}<$ $0.05)$, while the difference between groups $\mathrm{B}$ and $\mathrm{C}$ was not significant [25].

\section{CONCLUSIONS}

The exact pathophysiology of painful diabetic polyneuropathy is still unclear. Even analgetic therapy can never always completely treat the variety of patient's symptoms. Citicoline is one of the neuroprotector agents who has benefited from peripheral nerve lesion, as proved by some studies with animal trials. The use of citicoline may be recommended as adjuvant therapy, aside from analgetic and glycemic control. Further studies with a human sample are needed to assess the efficacy and safety of citicoline for painful diabetic polyneuropathy.

Conflict of interest: none declared Financial support: none declared

6. Nascimento OJM, Pupe CCB, Cavalcanti EBC. Diabetic neuropathy. Rev Dor. São Paulo. 2016;17(1):46-51.

7. Schreiber AK, Nones CFM, Reis RC, Chichorro JG, Cunha JM. Diabetic Neuropathic Pain: Physiopathology and Treatment. World Journal of Diabetes. 2015;6(3):432-44.

8. Mathebula SD. Polyol pathway: A possible mechanism of diabetes complications in the eye. African Vision Eye Health. 2015;74(1).

9. Callaghan BC, Cheng $\mathrm{H}$, Stables $\mathrm{CL}$, Smith AL, Feldman EL. Diabetic neuropathy: Clinical Manifestations and Current Treatments. Lancet Neurology. 2012;11(6):521-34.

10. Rajan RS, Gray L, George E. Painful diabetic neuropathy. Continuing Education in Anaesthesia, Critical Care \& Pain. 2014;14(5):230-5.

11. Mishra SC, Chhatbar KC, Kashikar A, Mehndiratta A. Diabetic Foot Clinical Updates. BMJ. 2017;359(1). 
12. Secades JJ. Citicoline: pharmacological and clinical review, 2016 update. Rev Neurol. 2017:1-75.

13. Doijad RC, Pathan AB, Pawar NB, Baraskar SS, Maske VD, Gaikwad SL. Therapeutic Applications of Citicoline and Piracetam as Fixed Dose Combination. Asian Journal of Biomedical and Pharmaceutical Sciences. 2012;2(12):15-20.

14. Qureshi SS, Gupta JK, Mishra P. Citicoline: A Potential Breakthrough in Cerebrovascular Disorder. Austin J Pharmacol Ther. 2016; 4(1):1-4.

15. Suyatna FD. Farmakologi Klinik Citicoline. CDK. 2010;7:360-1.

16. Deli G, Bosnyak E, Pusch G, Komoly S, Feher G. Diabetic Neuropathies: Diagnosis and Management. Neuroendocrinology. 2013;98:267-80

17. Emril DR, Wibowo S, Meliala L, Susilowati R. Cytidine 5'-diphosphocholine administration prevents peripheral neuropathic pain after sciatic nerve crush injury in rats. Journal of Pain Research. 2016;9:287-91.

18. Ozay R, Bekar A, Kocaeli H, Karl N, Filiz G, Ulus IH. Citicoline improves functional recovery, promotes nerve regeneration, and reduces postoperative scarring after peripheral nerve surgery in rats. Surgical Neurology. 2007;68:615-22.

19. Liu L, Zhenjun N, Hongbin H, Qingjun S, Xinbo J, Tianhou L, Jingjing P, Lingyao Z. Role of Citicoline in Promoting the Repair of Acute Peripheral Nerve Injury in Rat Models. Neuroscience and Biomedical Engineering. 2016;4(4):237-40.
20. Aslan E, Kocaeli H, Bekar H, Ulus IH. CDP-choline and its endogenous metabolites, Cytidine and choline, promote the nerve regeneration and improve the functional recovery of injured rat sciatic nerves. Neurological Research. 2011;33(7):766-73.

21. Caner B, Kafa MI, Bekar A, Kurt MA, Karl N, Cansev M, Ulus IH. Intraperitoneal administration of CDP-choline or a combination of Cytidine plus choline improves nerve regeneration and functional recovery in a rat model of sciatic nerve injury. Neurological Research. 2012;34(3):238.

22. Gundogdu EB, Bekar A, Turkyilmaz M, Gumus A, Kafa IM, Cansev M. CDP-choline modulates matrix metalloproteinases in rat sciatic injury. Journal of Surgical Research. 2016;200:655-63.

23. Zhang H, Chang M, Hansen CN, Basso DM, Noble-Haeusslein LJ. Role of matrix metalloproteinases and therapeutic benefits of their inhibition in spinal cord injury. Neurotherapeutics. 2011;8:206.

24. Kaplan T, Kafa IM, Cansev M, Bekar A, Karli N, Taskapilioglul MO, Kanar F. Investigation of the Dose-Dependency of Citicoline Effects on Nerve Regeneration and Functional Recovery in a Rat Model of Sciatic Nerve Injury. Turkish Neurosurgery. 2014;24(1):54-62.

25. Liu Y, Li N, Ran XW. Clinical effect of ligustrazine combined with citicoline for treatment of diabetic peripheral neuropathy. Zhongguo Zhong Xi Yi Jie He Za Zhi. 2008;28(7):606-9. 